\title{
Analysis of SWT-SVD Digital Image Watermarking Technique
}

\author{
Neha R.Sawant ${ }^{1}$, Pravin S. Patil ${ }^{2}$ \\ M.E. (Student), Electronics Engineering Department, SSVPS's BSD COE Dhule (MH) ${ }^{1}$ \\ Associate Professor \& Head of the Department of Electronics \& Communication Engineering, \\ SSVPS's BSD COE Dhule $(\mathrm{MH})^{2}$
}

\begin{abstract}
Last few years digital watermarking has facilitated the protection of copyright information through embedding the hidden information into the digital content. Digital image watermarking is one such the technology that has been developed to protect digital images from illegal manipulations. In this paper a new SWT-SVD semi-blind composite images watermarking algorithm that is robust against various attacks is presented .We use SWT transform to obtain 4-diffirent frequency images. Then we apply SVD on each subsection to modifying their singular value, on the basis of MSE \& PSNR values Experimental evaluation demonstrates that proposed algorithm is able to withstand variety of attacks as Salt \& Pepper noise, Rotation, Median filter, Vertical Mirroring, Horizontal Mirroring, Gaussian noise, Cropping, Contrast etc. Watermark is recovered efficiently.
\end{abstract}

Index Terms: Copyright protection, Blind watermarking, SWT (stationary wavelet transform), Singular value decomposition (SVD), MSE (mean square error), PSNR (peak signal to noise ratio).

\section{INTRODUCTION}

Digital watermarking is a technique which embeds The digital image watermarking scheme can be divided additional information called digital signature or into two categories. They are visible digital image watermark into the digital content in order to secure it. A watermarking and invisible image watermarking watermark is a hidden signal added to images that can be techniques. In visible watermarking, the information is detected or extracted later to make some affirmation about visible in the picture or video. Typically, the information the host image. The major point of digital watermarking is is text or a logo which identifies the owner of the original to find the balance among the aspects such as robustness document.

to various attacks, security and invisibility. The In invisible watermarking, information is added as digital invisibleness of watermarking technique is based on the data to audio, picture or video, but it cannot be perceived intensity of embedding watermark. Better invisibleness is as such. Further, the invisible watermarks are categorized achieved for less intensity watermark. So we must select into watermarking techniques as robust, fragile and semithe optimum intensity to embed watermark. In general fragile.

there is a little trade-off between the embedding strength (the watermark robustness) and quality (the watermark invisibility). Increased robustness requires a stronger embedding, which in turn increases the visual degradation of the images [1]. For a watermark to be effective, it should satisfy the following features. They are:

- Imperceptibility - It should be perceptually invisible so that data quality is not degraded and attackers are prevented from finding and deleting it. A watermark is called imperceptible if the watermarked content is perceptually equivalent to the original, UN watermarked content.

- Readily Extractable - The data owner or an independent control authority should easily extract it.

- Unambiguous - The watermark retrieval should unambiguously identify the data owner.

- Robustness - It should tolerate some of the common image processing attacks. A watermark is called robust if it resists a designated class of transformations.

- $\quad$ Robust watermarks may be used in copyright protection applications to carry copy and access control information.

Robust - Generally, a robust mark [2] is generally used for copyright protection and ownership identification because they are designed to withstand nearly all attacks such as loss compression, filtering operations and geometric distortions. These algorithms ensure that the image processing operations do not erase the embedded watermark signal.

- $\quad$ Fragile - In fragile techniques [3], even one bit change in image is not allowable. They are mainly applied to content authentication and integrity attestation, because they are sensitive to almost all modifications.

- $\quad$ Semi-fragile - Semi-fragile methods [4] and [5] are robust to incidental modifications such as JPEG compression, but fragile to other modifications such as high impact additive noises. That is, some incidental image manipulations have to be considered allowable during the process of media transmission and storage, while other malicious modifications (e.g. alteration of content) from attackers should be rejected. - Intentional distortion.

In this paper, we have introduced DWT-SVD technique to embed watermark image into the main or cover image, 
Vol. 5, Issue 12, December 2016

which proves robust to various kind of attacks which are mentioned later.

\section{RELATED BACKGROUND}

\section{A. Discrete Wavelet Transform}

The DWT decomposes input image into four components namely LL, HL, LH and $\mathrm{HH}$ where the first letter corresponds to applying either a low pass frequency operation or high pass frequency operation to the rows, and the second letter refers to the filter applied to the columns [6], which is shown in Fig.1. The lowest resolution level LL consists of the approximation part of the original image. The remaining three resolution levels consist of the detail parts and give the vertical high (LH), horizontal high (HL) and high $(\mathrm{HH})$ frequencies. In the proposed algorithm, watermark is embedded into the host image by modifying the coefficients of high-frequency bands i.e. HH sub band.

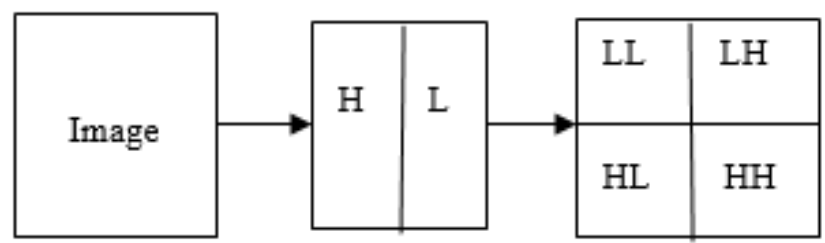

Figure1 DWT Transform

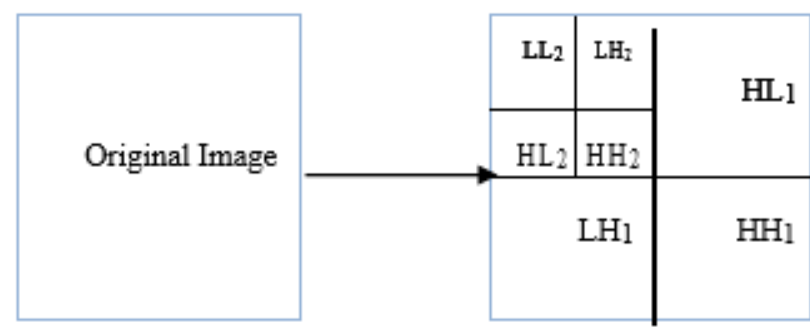

Figure 2 two level DWT decomposition

\section{B. Singular value Decomposition (SVD)}

SVD is one of the most powerful numeric analysis techniques with various applications including watermarking [7, 8 and 9]. SVD is a linear algebra technique used to solve many mathematical problems. Decompose a matrix that is not symmetric by considering a matrix $A$ which is of Dimension $m x n$ where $m \geq n$. The vectors in the expansion are the Eigen vectors of the square matrices $\mathrm{AA}^{\mathrm{T}}$ and $\mathrm{A}^{\mathrm{T}} \mathrm{A}$. The singular values are non-zero square roots of the square matrices $\mathrm{AA}^{\mathrm{T}}$ and $\mathrm{A}^{\mathrm{T}} \mathrm{A}$.

The singular value decomposition of A is given by,

(1)

$$
\text { A } \quad \text { USV } \quad \mathrm{T}
$$

Where $\mathrm{U}$ is an $\mathrm{m} \mathrm{x} \mathrm{m}$ real or complex unitary matrix and (the Conjugate transpose of $\mathrm{V}$ ) is an $\mathrm{n} \mathrm{x} \mathrm{n}$ real or complex unitary matrix such that,

$\begin{array}{ccccc}\mathrm{U} & \times & \mathrm{U}^{\mathrm{T}} & = & \mathrm{I} \\ \mathrm{V} & \times \mathrm{V} & \mathrm{T} & = & \mathrm{I}\end{array}$

Where, I represents an Identity matrix and $S$ is the diagonal matrix of order $\mathrm{m} \times \mathrm{n}$ having elements $\mathrm{S} 1, \mathrm{~S} 2$, $\mathrm{S} 3, \ldots . ., \mathrm{Sn}$.

Where,

$$
\mathrm{S} 1>\mathrm{S} 2>\ldots+\mathrm{Sn}
$$

The singular values of A are represented by the diagonal elements of $\mathrm{S}$. The columns of $\mathrm{U}$ matrix are known as the left singular values of $\mathrm{A}$ and the columns of $\mathrm{V}$ are known as the right singular values of A. Such a factorization is called the singular value decomposition of $\mathrm{A}$.

\section{PROPOSED METHOD}

Accurate segmentation of the images relies on the automated feature extraction methods that determine the best features to distinguish different tissues. Translation variant characteristics of (DWT) are its drawback. This leads it to extract remarkably different features from the same two images with only slight realignment [10]. Stationary wavelet transform [11] is used to overcome this problem by removing the down sampling procedure from the DWT and produces an over complete representation. Since all image are decomposed by SWT and the original images have the same size, SWT coefficients and textural features that are extracted from them can be used directly for segmentation without a need for projection [12].

\section{Stationary Wavelet Transform}

The Stationary wavelet transform (SWT) is similar to the DWT except the signal is never sub-sampled and instead the filters are up sampled at each level of decomposition. Figure. 3 shows flow of watermark Embedding and extraction \& validation process.

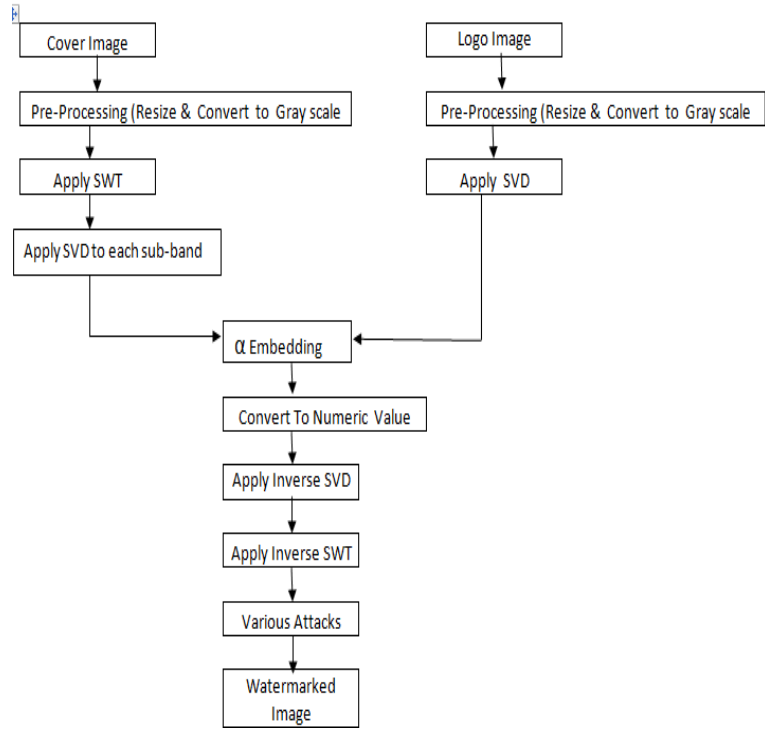


Vol. 5, Issue 12, December 2016

Figure. 3 Flow of watermark Embedding and extraction \& validation process

\section{Watermark Embedding}

1. First of all, we decompose the cover image into 4 sub-bands. In this paper, we use one level Haar transformation for decomposition of cover image A into 4 sub-bands [13].

2. After performing SWT, we perform SVD to each sub-band images i.e.,

$$
\mathrm{A}^{\mathrm{k}}=\mathrm{Ua}^{\mathrm{k}} \mathrm{Sa}^{\mathrm{k}} \mathrm{Va}^{\mathrm{KT}} \quad \mathrm{k}=1,2,3,4
$$

Where $\mathrm{k}$ denotes LL, LH, HL and HH sub-bands and $\lambda_{\mathrm{i}}^{\mathrm{K}}$, $\mathrm{i}=1, \mathrm{n}$ denotes the singular values of $\mathrm{Sa}^{\mathrm{k}}$.

3. In the same way, we apply SVD to watermark image, i.e.,

$$
\mathrm{W}=\mathrm{UwSwVw}^{\mathrm{T}}
$$

Where $\lambda w i, i=1, n$ denotes the singular values of $\mathrm{Sw}$.

4. After this, we modify the singular values of cover image in each sub-band with the singular values of watermark image, i.e.,

$$
\lambda \mathrm{i}^{* \mathrm{~K}}=\lambda \mathrm{i}^{\mathrm{K}}+\alpha \mathrm{k} \lambda \text { wi where } \mathrm{i}=1, \mathrm{n} \text { and } \mathrm{k}=1,2,3,4
$$

5. So, we obtain 4 sets of modified SWT coefficients, i.e.

$$
\mathrm{A} *^{\mathrm{K}}=\mathrm{Ua}^{\mathrm{K}} \mathrm{Sa}^{*}{ }^{\mathrm{K}} \mathrm{Va}^{\mathrm{KT}} \quad \text { where } \mathrm{k}=1,2,3,4
$$

6. Obtain the watermarked image $\mathrm{A}_{\mathrm{w}}$ by performing the ISWT using these 4 modified sub-bands.

\section{Watermark Extraction}

1. First of all, we use one-level Haar SWT to decompose watermarked (possibly distorted due to various kinds of attacks) image $A * k$ into 4 sub-bands.

2. Then, we apply SVD to each sub-band, i.e.

$$
\mathrm{A}^{* \mathrm{~K}}=\mathrm{Ua}^{\mathrm{K}} \mathrm{Sa}^{*}{ }^{\mathrm{K}} \mathrm{Va}^{\mathrm{KT}}, \mathrm{k}=1,2,3,4
$$

Where $\mathrm{k}$ denotes the attacked sub-band.

3. Then, we extract the singular values from each sub-band, i.e.

$$
\lambda w \mathrm{i}^{\mathrm{K}}=\left(\lambda \mathrm{i}^{\mathrm{K}}-\lambda \mathrm{i}^{\mathrm{K}}\right) / \alpha \mathrm{k} \text { where } \mathrm{i}=1, \mathrm{n} \text { and } \mathrm{k}=1,2,3,4 \text {. }
$$

4. Construct the four visual watermarks using the singular vectors, i.e.

$$
\mathrm{W}^{\mathrm{K}}=\mathrm{U}_{\mathrm{W}} \mathrm{S}_{\mathrm{W}} \mathrm{V}_{\mathrm{W}}^{\mathrm{T}}, \mathrm{k}=1,2,3,4
$$

\section{IV.EXPERIMENTAL RESULTS AND ANALYSIS}

The magnitudes of the singular values for each sub-band of the Lena image are shown in the fig. 4. Figure shows $512 \times 512$ gray scale cover image Lena, the $256 \times 256$ gray scale visual watermark copyright, the watermarked image, and the watermarks constructed from the four sub-bands. The scaling factor i.e. ${ }_{\mathrm{k}}$ for LL sub-band is taken to be 0.01 and 0.05 for other three sub-bands.

Our implemented scheme is based on the idea of replacing singular values of the $\mathrm{HH}$ band with the singular values of watermark. Below maximum and minimum singular values of all sub-bands of original image Lena is given. The wavelet coefficients are found to have largest value in LL band and lowest for HH band. Below figure 4- fig (a) Original cover, watermark \& watermarked Image, fig. (b) Extracted watermark images fig. (c) Plot of Singular values of Original Image fig. (d) Plot of Singular values of watermark Images fig. (e)Plot of Singular values watermarked Images.

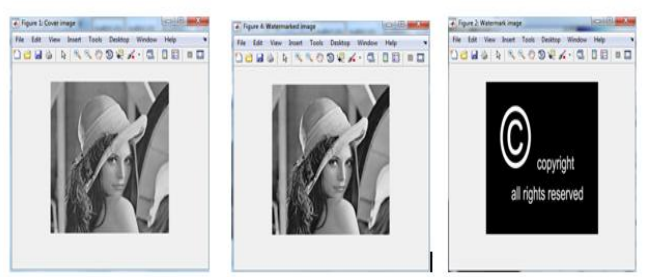

(a) Original cover, watermark \& watermarked Image
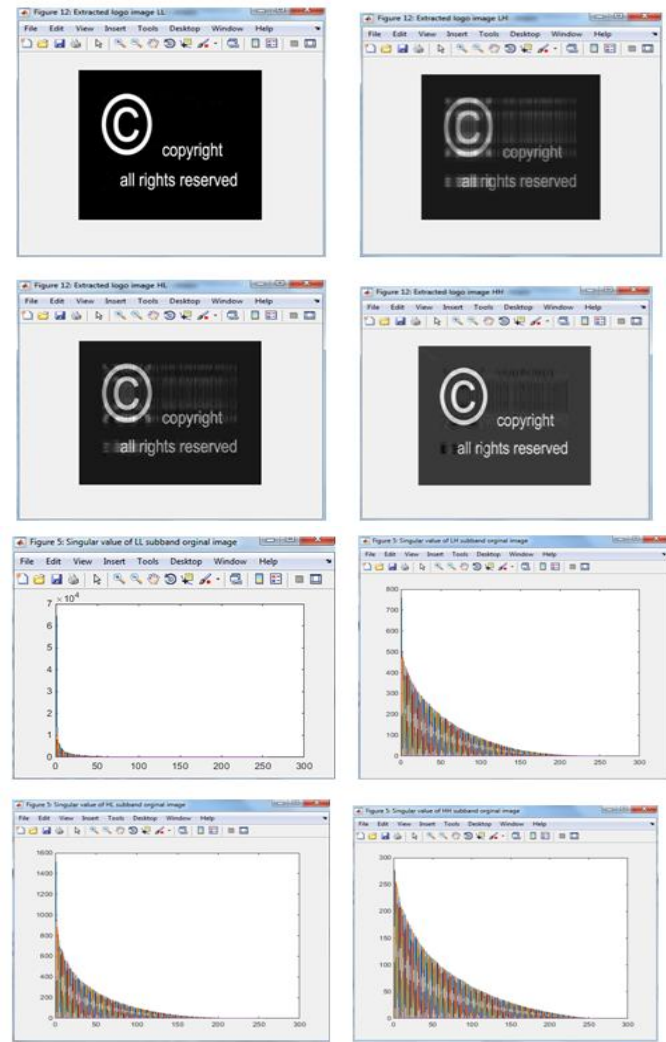

(b) Extracted watermark images from 4 sub bands Singular values of 4 sub-bands for Original Image 


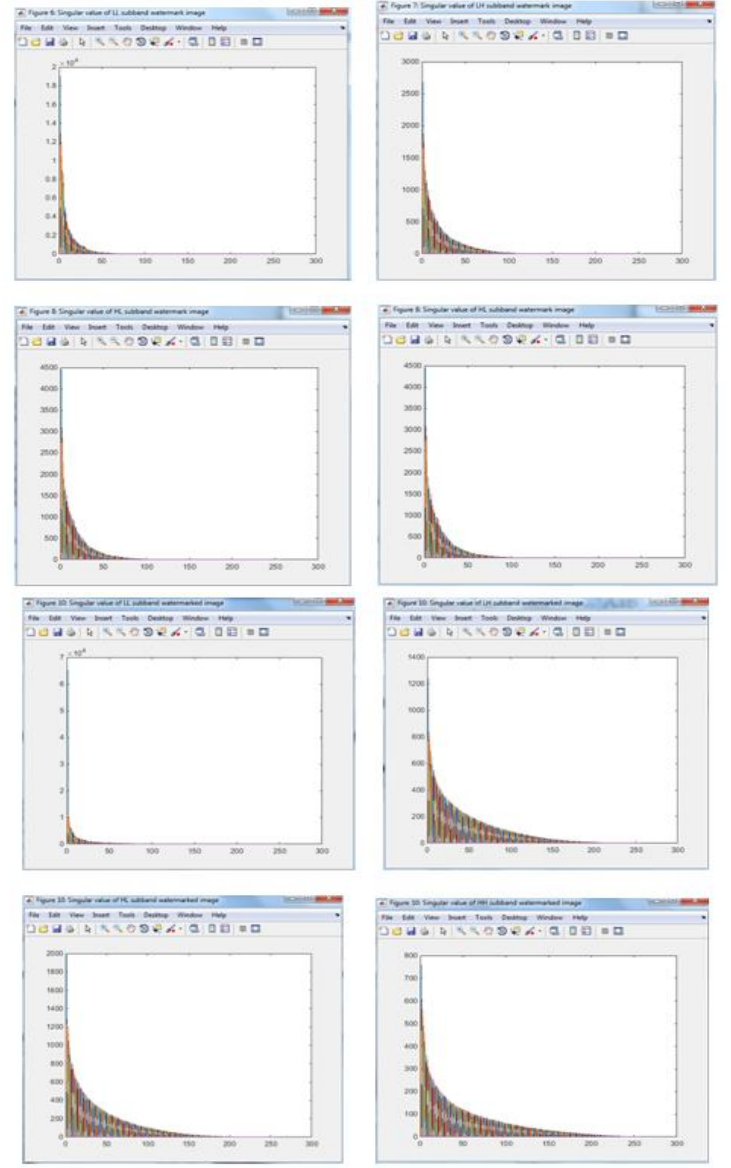

(d) Singular values of 4 sub-bands for watermark Image

(e) Singular values of 4 sub-bands for watermarked Image
Figure 4. (a) Original cover, watermark \& watermarked Image, (b) Extracted watermark images from 4 sub bands, (c) Singular values of 4 sub-bands for Original Image, (d) Singular values of 4 sub-bands for watermark Image and (e) Singular values of 4 sub-bands for watermarked Image.

\section{RESULT ANALYSIS}

To evaluate the performance of the watermarked images, there are some quality measures such as MSE (mean square error), PSNR (peak signal to noise ratio), and NCC (normalized cross correlation) [13].

$$
\begin{aligned}
& \begin{array}{l}
\mathrm{PSNR}=10 \log _{10}\left(\underline{255^{2}}\right. \\
\mathrm{MSE}
\end{array} \\
& \text { where } \\
& \left.\operatorname{MSE}=\left[\frac{1}{M \times N}\right]_{m=1}^{M} \underset{n=1}{M}(m, n)-I_{W}(m, n)\right]^{2} \\
& \text { and, } \\
& N C C=\frac{i \text { i w }(i, j) w^{*}(i, j)}{i i|w(i, j)|^{2}}
\end{aligned}
$$

After analysis with DWT-SVD our proposed method using SWT-SVD Gives high PSNR Values as shown in below table, More accurate., High robustness.

Table 1. PSNR values for sub-band of extracted watermark image using DWT SVD

\begin{tabular}{|c|c|c|c|c|c|}
\hline \multicolumn{5}{|c|}{ PSNR values for sub-band of extracted watermark image using DWT SVD } \\
\hline S.No & Type of noise & LL & LH & HL & HH \\
\hline 1 & Salt \& Pepper noise & 3.09 & 4.43 & 4.43 & 4.43 \\
\hline 2 & Rotation $50^{\circ}$ & 13.08 & 25.99 & 19.46 & 29.94 \\
\hline 3 & Median filter & 19.41 & 20.27 & 17.96 & 23.77 \\
\hline 4 & Vertical Mirroring & 40.02 & 24.65 & 25.17 & 23.57 \\
\hline 5 & Horizontal Mirroring & 40.02 & 24.65 & 25.19 & 23.57 \\
\hline 6 & Gaussian noise & 5.06 & 4.43 & 4.43 & 4.43 \\
\hline 7 & Cropping & 1.75 & 14.32 & 10.73 & 16.01 \\
\hline 8 & Contrast & 3.77 & 19.4 & 15.91 & 22.3 \\
\hline
\end{tabular}

Table 2. PSNR values for sub-band of extracted watermark image using SWT SVD

\begin{tabular}{|c|c|c|c|c|c|}
\hline \multicolumn{5}{|c|}{ PSNR values for sub-band of extracted watermark image using SWT SVD } \\
\hline S.No & Type of noise & LL & LH & HL & HH \\
\hline 1 & Salt \& Pepper noise & 36.63 & 46.45 & 48.24 & 48.53 \\
\hline 2 & Rotation $50^{\circ}$ & 17.65 & 17.63 & 17.63 & 17.63 \\
\hline 3 & Median filter & 37.18 & 40 & 39.82 & 40.49 \\
\hline 4 & Vertical Mirroring & 34.82 & 44.67 & 37.58 & 50.49 \\
\hline 5 & Horizontal Mirroring & 34.28 & 36.59 & 40.82 & 44.67 \\
\hline 6 & Gaussian noise & 35.37 & 42.6 & 42.87 & 41.01 \\
\hline 7 & Cropping & 26.11 & 26.04 & 26.04 & 26.04 \\
\hline 8 & Contrast & 31.68 & 31.16 & 30.93 & 31.21 \\
\hline
\end{tabular}


Vol. 5, Issue 12, December 2016
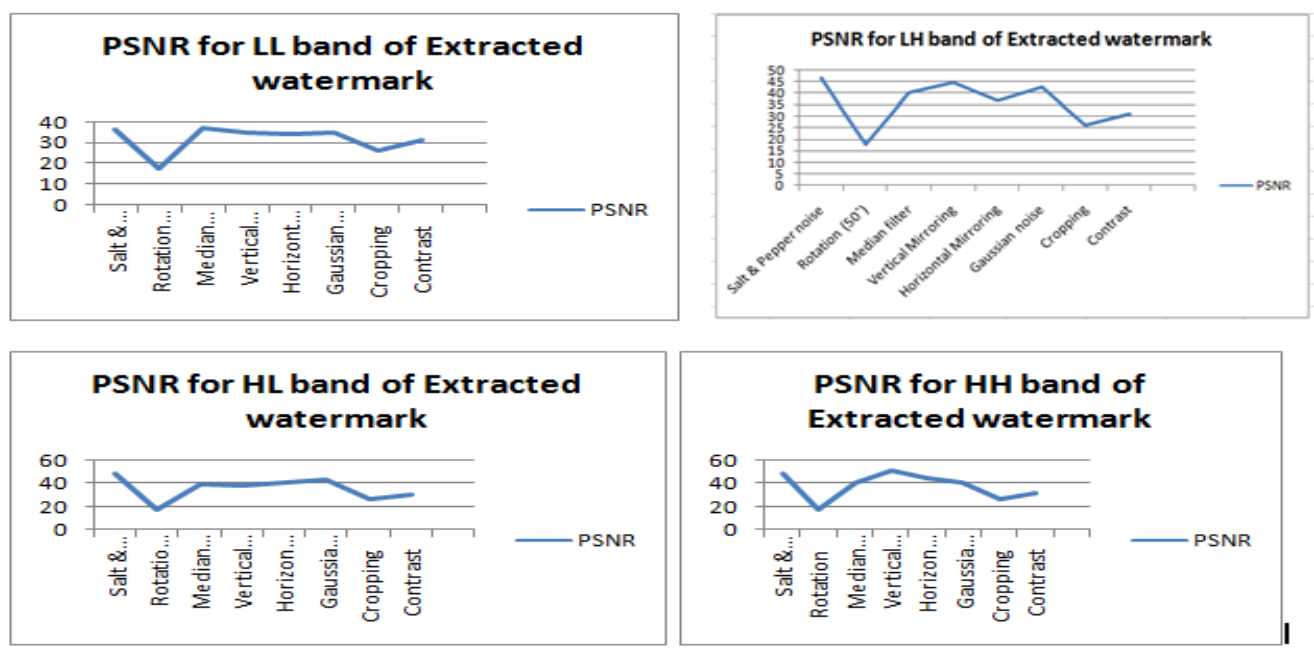

Figure 5. PSNR values of 4 sub-band extracted watermark image

\section{CONCLUSION}

In this paper, a hybrid image-watermarking technique based on SWT and SVD has been presented, where the watermark is embedded on the singular values of the cover image's SWT sub bands. The technique fully exploits the respective feature of these two transform domain methods: spatio-frequency localization of SWT and SVD efficiently represents intrinsic algebraic properties of an image. Experimental results of the proposed technique have shown both the significant improvement in imperceptibility and the robustness under attacks. Further work of integrating the human visual system characteristics into our approach is in progress.

\section{REFERENCES}

[1] Dr. M. Mohamed Sathik and S. S. Sujatha "Authentication of Digital images by using a semi-fragile watermarking technique", IJARCSSE Trans, vol. 2, pp.39-44(2012).

[2] Ram Kumar M and Akansu N, "A Robust Protocol for Providing Ownership of Multimedia content", IEEE trans on Multimedia, Vol.6, pp.469-478 (2004).

[3] Celik,M.U., Sharma, G., Saber E. and Tekalp, A.M., "Hierarchical Watermarking for Secure Image Authentication with Localization, "IEEE Trans on Image Processing, Vol.11, pp.585-595(2002).

[4] Lin.C, Su.T and Hsieh.W, "Semi-Fragile Watermarking Scheme for Authentication of JPEG Images", Tamkang Journal of Science and Engineering, Vol.10, No.1, pp.57-66 (2007)

[5] Zhou.X, Duan X., and Wang D., "A Semi-fragile Watermark Scheme for Image Authentication", IEEE International Conference on Multimedia modelling, pp.374-377 (2004).

[6] Xiang-Gen Xia, Charles G.Boncelet, and Gonzalo: Wavelet Transform based watermark for digital images. In: OPTICS EXPRESS, 1998 Vol.3, No.12, pp 497-511.

[7] B. Jagadeesh, S. Srinivas Kumar and K. Raja Rajeswari, "A Genetic Algorithm Based Oblivious Image Watermarking Scheme using Singular Value Decomposition (SVD)", 2009.

[8] N. V. Dharwadkar, B. B. Amberker and A.Gorai, "Non-blind Watermarking scheme for colour images in RGB space using DWT-SVD”, Proceedings IEEE, Vol. 04, November 2011.

[9] S. Kumar, A. Kumar Saini and P. Kumar, "SVD based Robust Image digital watermarking using DWT", IEEE, Vol. 45, 2012.

[10] Y. Zhang, Z. Dong, L. Wu, S. Wang, Z. Zhou, "Feature extraction of brain MRI by stationary wavelet transform", Proc. Int. Conf. Biomed. Eng. Compute. Sci., pp. 1-4, 2010.

[11] M. Unser, "Texture classification and segmentation using wavelet frames", IEEE Trans. Image Process., vol. 4, no. 11, pp. 15491650, 1995.
[12] A. Demirhan, İ. Güler, "Combining stationary wavelet transform and self-organizing maps for brain MR image segmentation", Eng. Appl. Artif. Intel. vol. 24, pp. 358-367, 2011.

[13] Emir Ganic, and Ahmet M. Eskicioglu, "Robust DWT-SVD Domain Image Watermarking: Embedding date in All Frequencies," CiteSeerX, MM\&SEC'04, September 20-21, 2004, Magdeburg, Germany. 